\title{
Three Sites and You Are Out: Ternary Synergistic Allostery Controls Aromatic Amino Acid Biosynthesis in Mycobacterium tuberculosis
}

\author{
Nicola J. Blackmore ${ }^{1}$, Sebastian Reichau ${ }^{1}$, Wanting Jiao ${ }^{1}$, Richard D. Hutton ${ }^{1}$, Edward N. Baker ${ }^{2}$, Geoffrey B. Jameson ${ }^{3}$ \\ and Emily J. Parker ${ }^{1}$
}

1 - Biomolecular Interaction Centre, Department of Chemistry, University of Canterbury, Private Bag 4800, Christchurch 8140 , New Zealand

2 - Maurice Wilkins Centre for Molecular Biodiscovery, School of Biological Sciences, University of Auckland, PO Box 92019, Auckland 1142, New Zealand

3 - Riddet Institute and Centre for Structural Biology, Institute of Fundamental Sciences, Massey University,

PO Box 11 222, Palmerston North 4442, New Zealand

Correspondence to Emily J. Parker: Department of Chemistry, University of Canterbury, Private Bag 4800, Christchurch, New Zealand. emily.parker@canterbury.ac.nz

http://dx.doi.org/10.1016/j.jmb.2012.12.019

Edited by S. Edelstein

\begin{abstract}
3-Deoxy-D-arabino-heptulosonate 7-phosphate synthase (DAH7PS) catalyzes the first step in the shikimate pathway, the pathway responsible for the biosynthesis of the aromatic amino acids Trp, Phe, and Tyr. Unlike many other organisms that produce up to three isozymes, each feedback-regulated by one of the aromatic amino acid pathway end products, Mycobacterium tuberculosis expresses a single DAH7PS enzyme that can be controlled by combinations of aromatic amino acids. This study shows that the synergistic inhibition of this enzyme by a combination of Trp and Phe can be significantly augmented by the addition of Tyr. We used X-ray crystallography, mutagenesis, and isothermal titration calorimetry studies to show that DAH7PS from $M$. tuberculosis possesses a Tyr-selective site in addition to the Trp and Phe sites, revealing an unusual and highly sophisticated network of three synergistic allosteric sites on one enzyme. This ternary inhibitory response, by a combination of all three aromatic amino acids, allows a tunable response of the protein to changing metabolic demands.
\end{abstract}

(C) 2013 Elsevier Ltd. All rights reserved.

\section{Introduction}

Allosteric regulation of protein functions is critical for the control of metabolism. Allostery is the process by which the binding of one or more effectors to a protein causes a functional response at a distant site. Allostery is widely observed in biological systems and is crucial in signal transduction, metabolism, catalysis, and gene regulation. ${ }^{1-5}$

Allostery arises from a ligand-induced perturbation in the population distribution between different forms of a protein. Protein molecules exist as ensembles of different states and the population distribution of these ensembles is defined by the free-energy landscape of the particular protein system. ${ }^{3}$ Early examples of allosteric regulation mostly featured large changes in the average conformation of the ensemble in oligomeric proteins (mainly enthalpy driven). Such protein systems include hemoglobin and aspartate transcarbamoylase. ${ }^{6,7}$ The wellknown Monod-Wyman-Changeux model of allostery (also known as the concerted model) has been widely applied to explain the behavior of these allosteric systems. ${ }^{6}$ More recently, allostery associated with more subtle changes in protein structure has been investigated. Binding of an allosteric effector may alter the free-energy landscape of the protein so that allostery is mediated by changing the dynamic fluctuations about the average conformation of the protein ensemble (mainly entropy 

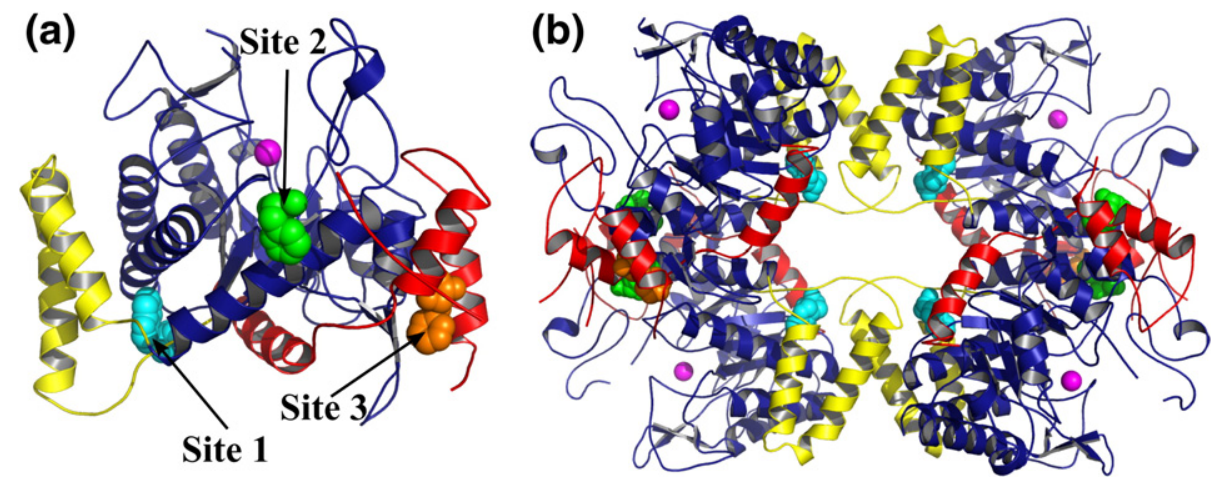

Fig. 1. (a) Monomer structure of MtuDAH7PS in complex with Phe and Trp (PDB 3KGF). Sites 1, 2, and 3 are labeled. (b) Homotetramer of MtuDAH7PS in complex with Phe and Trp (PDB 3KGF). The N-terminal extra-barrel elements (the $\mathrm{N}$-termini and $\alpha 0 \mathrm{a}-\alpha 0 \mathrm{c}$ helices) are shown in red, and the additional $\alpha 2 \mathrm{a}, \alpha 2 b$ helices are shown in yellow. Active-site metal ions are shown as purple spheres. The Trp binding in site 1 is shown as cyan spheres, the Phe in site 2 is shown as green spheres, and the Phe ligand in site 3 is shown as orange spheres.

driven). ${ }^{8-13}$ Both allosteric regulation in monomeric proteins and purely entropy-driven allostery have been described. ${ }^{14-16}$ The role of protein dynamics in determining function is becoming more recognized, contributing to the increase in our understanding of protein function and regulation. ${ }^{8-13}$

The enzyme 3-deoxy-D-arabino-heptulosonate 7phosphate synthase (DAH7PS) catalyzes the first reaction in the shikimate pathway, the pathway responsible for the biosynthesis of the aromatic amino acids Trp, Phe, and Tyr. ${ }^{17}$ This pathway is present in microorganisms ${ }^{18,19}$ but absent in higher organisms, making the enzymes in this pathway good targets for antimicrobial drug development. DAH7PS catalyzes the aldol reaction between phosphoenolpyruvate (PEP) and erythrose 4-phosphate (E4P) to produce 3-deoxy-D-arabino-heptulosonate 7-phosphate, which is converted to chorismate via several enzyme-catalyzed steps. At this point, the pathway branches: one branch produces Phe and Tyr, and the other produces Trp. As the first enzyme in a branched pathway, DAH7PS is a major control point for the shikimate pathway and the regulation of this enzyme is essential for metabolic control.

Previous studies have shown that the DAH7PS enzyme from Mycobacterium tuberculosis (MtuDAH7PS) is feedback-regulated by the pathway end products Trp, Phe, and Tyr by means of a sophisticated mechanism of allosteric regulation. ${ }^{20}$ Dual combinations of Trp and Phe (or Trp and Tyr to a lesser extent) show an inhibitory effect on MtuDAH7PS, whereas Trp, Phe, or Tyr alone results in limited inhibition. Crystal structures of ligand-free MtuDAH7PS have been solved and showed that the quaternary structure of this enzyme is a homotetramer of $(\beta / \alpha)_{8}$ TIM barrel subunits. The dimer and tetramer interfaces are formed by accessory elements to the basic barrel. Crystal structures of Phe+Trp-bound MtuDAH7PS (Fig. 1b) show that
Phe and Trp bind in distinct allosteric sites in the dimer and tetramer interfaces, respectively, about $20 \AA$ away from each other and over $20 \AA$ away from the active site. Furthermore, comparison with the ligand-free MtuDAH7PS structure reveals that there is no change in gross conformation of the enzyme upon binding of allosteric effectors (Trp + Phe). ${ }^{20}$ Studies using molecular dynamics simulations have shown that the allostery observed in MtuDAH7PS upon Trp+Phe binding is mainly entropy driven and that inhibition is achieved by the reduction in affinity of the enzyme for its substrate E4P. ${ }^{21}$

The central role that MtuDAH7PS plays in the regulation of aromatic amino acid biosynthesis has been further highlighted by the observation that MtuDAH7PS forms a complex with $M$. tuberculosis chorismate mutase, the branch point enzyme for Tyr and Phe biosynthesis. ${ }^{22}$ Formation of this complex results in the activation of the chorismate mutase and confers regulatory properties to this enzyme, with no effect on the activity of MtuDAH7PS.

Here, we report the first-time observation of a ternary response of MtuDAH7PS to all three aromatic amino acids (Trp+Phe+Tyr). We demonstrate that the binary inhibitory response in MtuDAH7PS caused by Trp and Phe is augmented by the binding of Tyr to an independent site on the enzyme. As a result, the catalytic activity of MtuDAH7PS is completely abolished when all three aromatic amino acids are present. This fine-tuning of enzyme activity by three different allosteric effectors with three distinct binding sites is, to our knowledge, unprecedented and provides an important example of an extremely sophisticated and novel mechanism in protein allosteric regulation. These findings may have implications for the development of antimycobacterial drugs targeting the allosteric sites of this essential protein. 


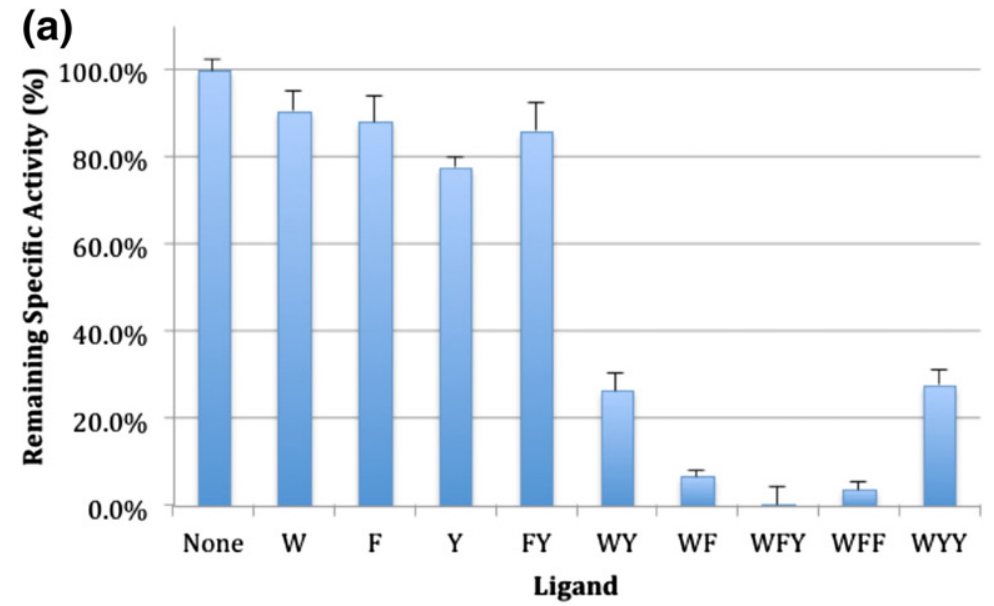

(b)

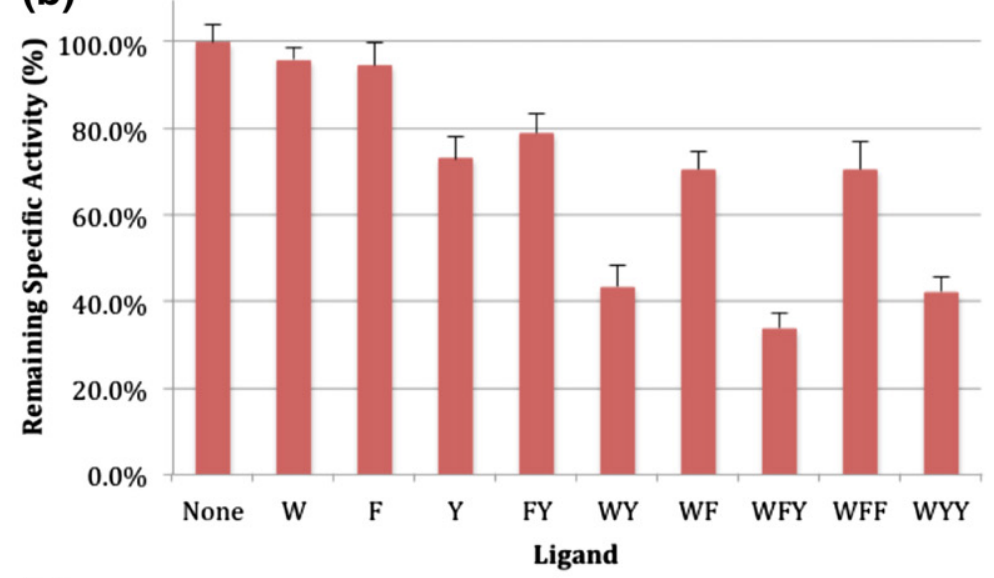

(c)

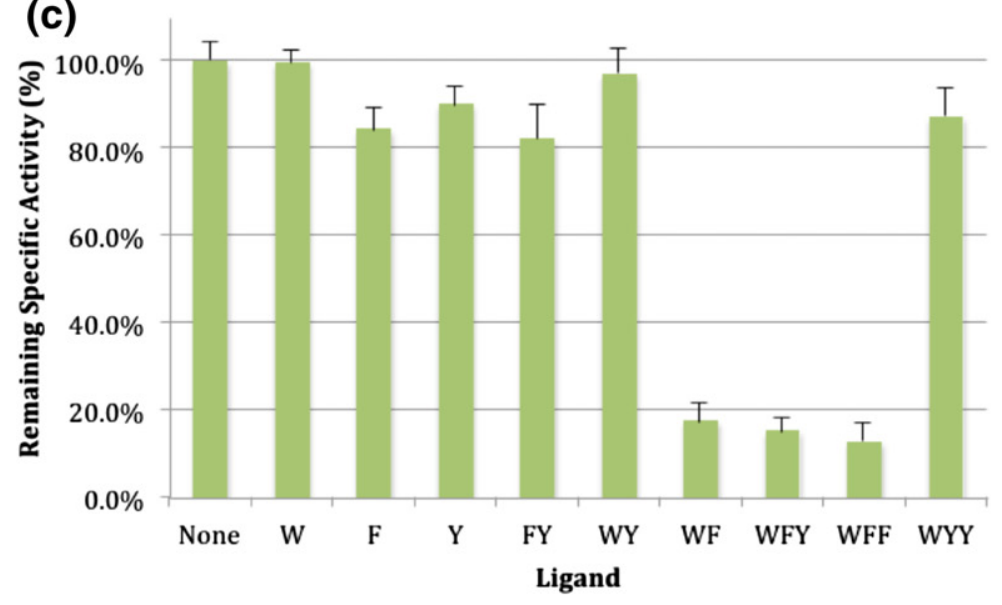

Fig. 2. Influence of single aromatic amino acids and combinations of aromatic amino acids on the specific activity of wild-type MtuDAH7PS and its mutants. (a) MtuDAH7PS wild type. (b) MtuDAH7PS R171A. (c) MtuDAH7PS R256A. Each ligand is present at a concentration of $200 \mu \mathrm{M}$ and is denoted by the single-letter amino acid code (W, Trp; F, Phe; Y, Tyr). The combinations $F Y, W Y, W F$, and WFY refer to each amino acid being present at a concentration of $200 \mu \mathrm{M}$, and WFF and WYY refer to the presence of $200 \mu \mathrm{M}$ Trp with either $400 \mu \mathrm{M}$ Phe or Tyr.

\section{Results}

Wild-type MtuDAH7PS is potently inhibited by
combination of all three aromatic amino acids

The catalytic activity of MtuDAH7PS was tested in the presence of aromatic amino acids Trp, Phe, and Tyr (Fig. 2a). In the presence of any single aromatic amino acid, no significant loss of activity is observed relative to the activity observed in the absence of added aromatic amino acids. However, binary combinations, particularly those involving Trp (Trp + Phe, Trp + Tyr), show significant inhibition, as previously reported. ${ }^{20}$ The most potent binary combination, Trp + Phe, reduces the activity of the wild-type MtuDAH7PS to 7\% (Fig. 2a). Interestingly, the inhibition observed from Trp + Phe is further augmented by the addition of Tyr, and this ternary combination of all three aromatic amino acids almost 
completely eliminates MtuDAH7PS catalytic activity (reduced to $1 \%$ ). In order to investigate whether this inhibition was attributable specifically to the presence of all three amino acids, rather than to concentration effects of binary combinations, we tested different binary combinations of aromatic amino acids (WFF and WYY in Fig. 2a). The inhibition observed in the ternary combination $(T r p+P h e+T y r)$ exceeds the effects that arise from the equivalent binary combinations of $\operatorname{Trp}+$ Phe or Trp + Tyr (WFF or WYY), indicating that Tyr specifically augments the inhibition of this enzyme by Trp and Phe.

\section{Crystal structures of DAH7PS reveal three distinct aromatic amino acid binding sites}

Intrigued by the discovery of inhibition of MtuDAH7PS by a ternary combination of Trp + Phe + Tyr, we sought structural information on the location of the Tyr binding site. Previous structural studies have identified three distinct sites for aromatic amino acid binding on MtuDAH7PS (Fig. 1a). ${ }^{20}$ Site 1 is located in the tetramer interface and made up mostly from residues of the $\alpha 2 \beta 3$ loop extension and helix $\alpha 1$. Site 1 is occupied by Trp. Site 2 is located in the dimer interface, capped by the $\beta 0$ sheet of the $\mathrm{N}$ terminal barrel extension. Site 2 is occupied by Phe when Phe and Trp are included in the crystallization conditions or soaked into the crystals. Site 3 is located between the extra-barrel helices at the $\mathrm{N}$ terminus (helices $\mathrm{aOa}$ and $\mathrm{\alpha Ob}$ ) and the $\alpha 3$ helix of the main barrel (Fig. 1a). This site is filled in just two of the four subunits of the biological unit. This site was found to be occupied by Phe, in addition to site 2 , when crystals were soaked in high concentrations (2 mM) of Trp and Phe. When lower ligand concentrations for co-crystallization were employed (100 $\mu \mathrm{M}$ Trp and Phe), site 3 is unoccupied. ${ }^{21}$ As these concentrations for co-crystallization were similar to those required for the inhibition by Trp + Phe, site 1 and site 2 were identified as the relevant sites for the inhibitory response to the binary combinations of Trp and Phe. Moreover, mutation of site 3 did not alter the inhibition of the enzyme by Trp and Phe. ${ }^{20}$ These observations left the role of site 3 unclear. Therefore, extensive screening of crystal soaking conditions was conducted in order to further elucidate the ligand preferences of the respective sites and to locate possible Tyr binding sites.

Tyr shows promiscuous binding to both site 2 and site 3

The structure of MtuDAH7PS in complex with only Tyr was determined at $2.3 \AA$ resolution from a crystal soaked in a solution containing Tyr at a concentration of $330 \mu \mathrm{M}\left(R_{\text {free }}=0.164\right.$, Table S2). The overall conformation of the protein is virtually unchanged by the presence of Tyr, with the rmsd between the unliganded [Protein Data Bank (PDB) code 3NV8] and Tyr-bound (PDB code 2YPP) structures being $0.240 \AA$ (for 5033 atoms). After refinement of the structure, clear continuous electron density corresponding to Tyr was found in both site 2 and site 3 (Fig. S2 and Fig. S3). Tyr occupies site 2 in both sites of the dimeric asymmetric unit (i.e., in all four sites of the functional tetramer), whereas site 3 is only occupied by Tyr in two of the four subunits, as had been earlier observed for Phe. ${ }^{20}$ The soaking of crystals in solutions containing lower concentrations of Tyr resulted in structures that either had no Tyr bound or partially defined electron density corresponding to Tyr in both site 2 and site 3 . Thus, Tyr shows promiscuous binding to these two sites, but it does not bind to site 1 , in contrast to that observed at high concentrations of just Phe (PDB code 3NUD).

In order to establish the binding sites involved in the inhibition by the Trp+Tyr combination, we obtained the crystal structure of MtuDAH7PS in complex with both Trp and Tyr by soaking crystals in a solution containing Trp and Tyr $(2 \mathrm{mM}$ concentration of each ligand). In the Trp + Tyr-soaked structure (2.76 $\AA, R_{\text {free }}=0.25$, PDB code 2YPQ), Trp occupies site 1 and, as observed in the Tyr-only structure, sites 2 and 3 are occupied by Tyr. The binding mode of Trp is consistent with the previously reported structures (PDB codes 3NUE, 3RZI, and 3KGF) and the Tyr binding mode is consistent with that found in the Tyr-only soaked structure.

\section{Tyr binding mode in site 2}

Site 2 is located in the dimer interface and the Tyr in this position interacts with residues from both subunits (Fig. 3a). The Tyr side chain binds in a pocket mostly formed by hydrophobic residues (Trp3, Tyr173*, Ala174*, and Ala174, where * indicates residues from the other subunit). The carboxylate of Tyr forms hydrogen bonds to the amide group of Asn175, a water molecule, which in turn interacts with the indole $\mathrm{N}-\mathrm{H}$ of Trp3, and a salt bridge to the guanidinium moiety of Arg171. The amino group of Tyr forms hydrogen bonds to the backbone carbonyl of Phe91, the side-chain carbonyl of Asn175 and, mediated through a water molecule, the main-chain carbonyl group of Asn94. The two Tyr ligands in different subunits are relatively close to each other, with the distance between their hydroxyl groups being $4.8 \AA$. The binding mode of Tyr in site 2 is very similar to the way Phe was observed to bind to this site (see also Fig. S5), the amino acid moieties of Tyr and Phe establish the same contacts to the protein, and there are no elements on the proteins that seem to be predisposed for the recognition of the Tyr hydroxyl group, albeit the addition of this moiety can be accommodated in the binding site. 


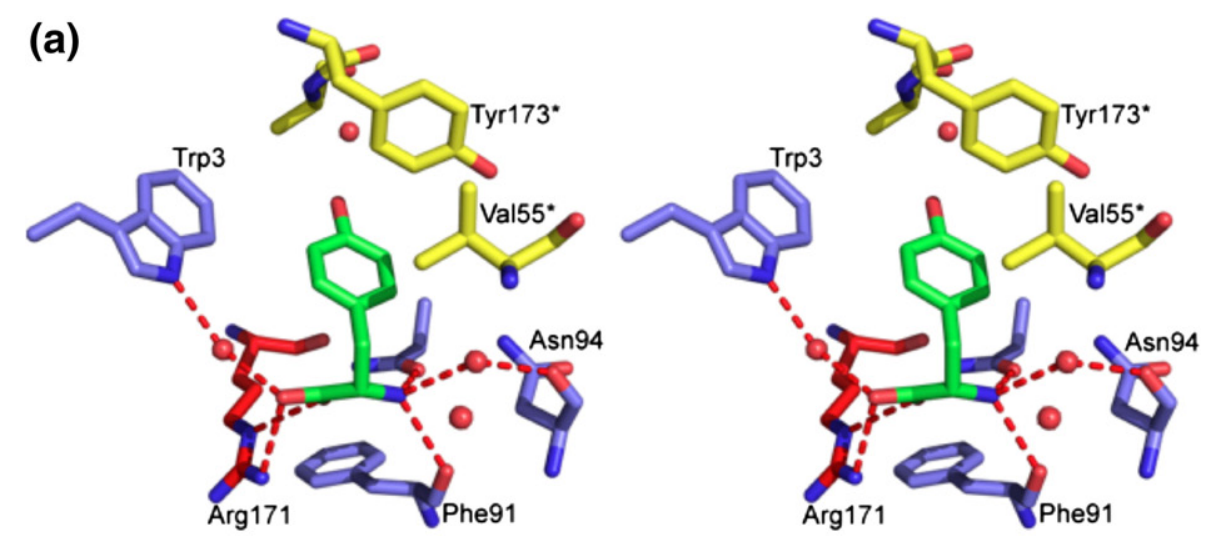

(b)
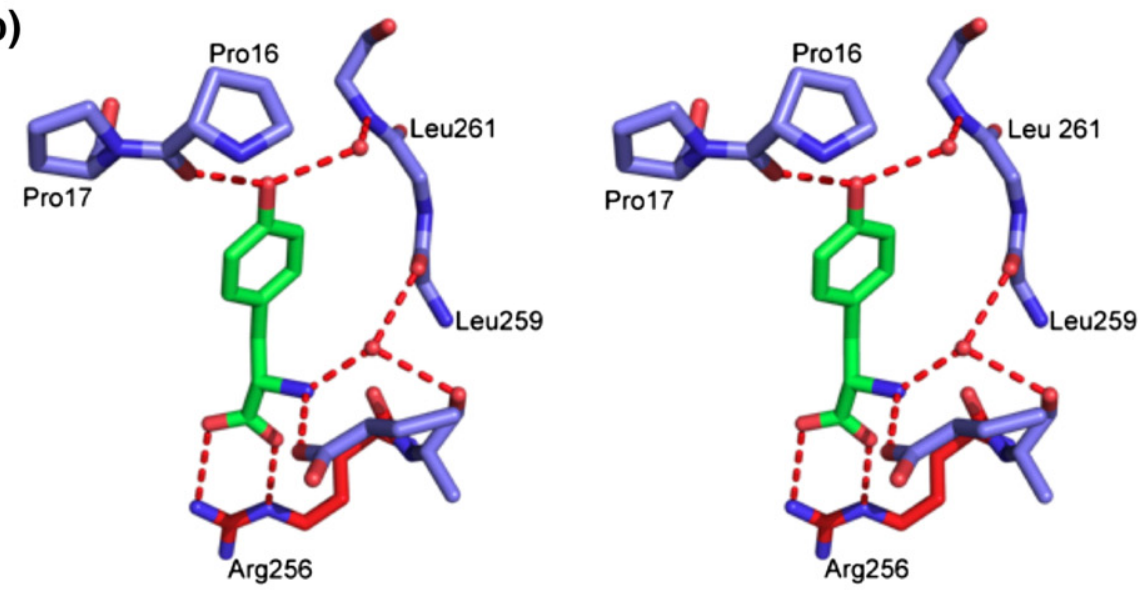

(c)
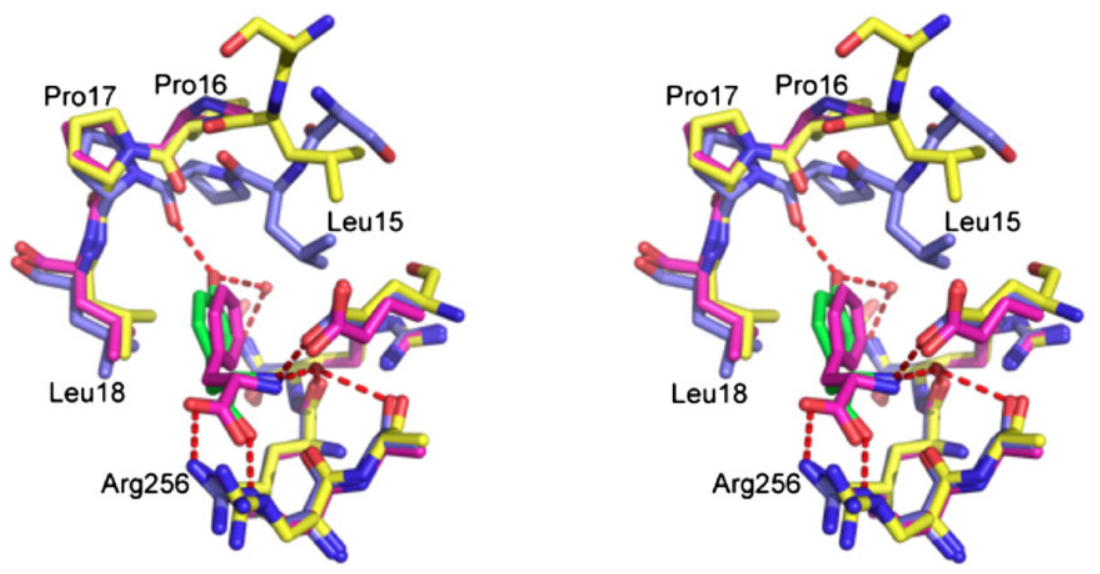

Fig. 3. Stereo diagrams showing the binding of Tyr in site 2 and Tyr and Phe in site 3. (a) Tyr binding in site 2 of subunit $B$ is shown with yellow carbons; residues from subunit $A$ are shown with blue carbons, and residues from subunit $B$, except Arg171, are shown with red carbons (PDB code 2YPP). (b) Tyr binding in site 3 of subunit B is shown with blue carbons, and residues from subunit $B$ are shown with green carbons, except for Arg256, which is shown with red carbons. Two water molecules mediating contacts between the Tyr ligand and the enzyme are shown as red spheres (PDB code 2YPP). (c) Overlay showing the conformational change induced by binding of Tyr in site 3. In the unliganded (yellow carbons, PDB code 3NV8) and Phe-liganded (purple carbons, PDB code 3NUD) structures, the loop containing Pro16 and Pro17 is in an open conformation and, in the case of the Phe-bound structure, also poorly defined. In the Tyr-bound structure (blue carbons, PDB code 2YPP), the carbonyl of Pro16 forms a hydrogen bond to the Tyr phenolic hydroxyl, causing a conformational change in the loop containing Pro16. A very similar arrangement is observed in the Trp + Tyr-bound structure (PDB code 2YPQ). 


\section{Tyr binding mode in site 3}

In contrast to Tyr binding in site 2, specific interactions with the phenolic hydroxyl of Tyr are established when Tyr binds in site 3: the main-chain carbonyl of Pro16 and a water molecule, which in turn interacts with the backbone amide $\mathrm{N}-\mathrm{H}$ of Leu261, are located 2.7 and $2.9 \AA$ from the phenolic oxygen, respectively. The carboxylate of Tyr interacts with the guanidinium group of Arg256. The amino group forms a salt bridge with the carboxylate of Glu53 and a hydrogen bond to a water molecule, which in turn makes contacts with the backbone carbonyls of Leu259 and Ala257 (Fig. 3b). Comparing the binding mode of Phe to that of Tyr in this site reveals that the amino acid moieties of both ligands interact in a very similar manner with the binding site (Fig. 3c). The phenyl ring of both the Tyr and Phe ligands also show similar orientations in both structures. However, due to its phenolic hydroxyl group, Tyr is able to establish an additional hydrogen bond to Pro16.

While the overall conformation of the enzyme is hardly affected by the presence of Tyr, several subtle conformational changes occur in the $\beta 0 a 0$ loop comprising residues 10-18 near site 3 (Fig. 3c). The amide nitrogen of Pro16 and its carbonyl group are shifted by $2.2 \AA$ and $1.4 \AA$, respectively, in the presence of Tyr, enabling a hydrogen bond interaction to be formed between the Pro16 carbonyl and the Tyr hydroxyl group. In the absence of ligand or in the presence of Phe in site 3 , the $\beta 0 \alpha 0$ loop adopts a more open conformation or is disordered.

\section{Phe at low concentration preferentially binds to site 2}

A new structure of MtuDAH7PS in complex with Phe at $2.0 \AA$ resolution was obtained by soaking crystals for a limited time $(1 \mathrm{~h})$ in a solution containing a lower concentration ( $200 \mu \mathrm{M}$ compared to the previously used $2 \mathrm{mM}$ ) of Phe (Tables S1 and S2, PDB code 2YPO, $\left.R_{\text {free }}=0.159\right)$. The overall structure obtained by this method is very similar to that obtained using the higher soaking concentration of Phe (PDB code 3NUD, rmsd=0.329 $\AA$ for 4533 atoms). However, in the crystals obtained at the lower Phe concentration, the only ligand site that showed clear continuous electron density corresponding to Phe is site 2 (Fig. S1). This observation confirms that site 2 is indeed the preferred binding site for Phe. The binding mode of Phe in this new structure is identical to the binding mode previously observed. ${ }^{20}$

\section{Amino acid substitutions at sites 2 and 3 reveal allosteric site ligand selectivities}

In order to further investigate the selectivity of sites 2 and 3 for Phe and Tyr, we made substitutions to
Ala for key Arg residues involved in ligand binding in both site 2 at the dimer interface (R171, red carbons in Fig. 3a) and site 3 (R256, red carbons in Fig. 3b). These mutations did not affect protein folding (as shown by circular dichroism spectrophotometry, Fig. S4, Supplementary Material), and both mutant enzymes were catalytically competent (full kinetic characterization is provided in the Supplementary Material, Table S3).

\section{Mutation of Arg171 (at site 2) significantly impairs Phe binding}

Arg171 forms a salt bridge with the carboxylate functionality of Phe or Tyr at site 2. In contrast to the wild-type enzyme, the activity of R171AMtuDAH7PS is only slightly inhibited by Trp+Phe (Fig. 2b). On the other hand, R171AMtuDAH7PS retains significant sensitivity to the combination of Trp and Tyr, with specific activity reduced to $\sim 40 \%$ of that observed for this mutant in the absence of aromatic amino acids (Fig. 2b). In the presence of all three ligands, Trp, Phe, and Tyr, the R171AMtuDAH7PS activity is similar to that observed in the presence of Trp and Tyr. To further probe ligand binding, isothermal titration calorimetry (ITC) was used to determine the dissociation constants for Tyr and Phe (Table 1). Phe and Tyr both bind to wild-type MtuDAH7PS with similar affinities $\left(K_{\mathrm{d}} \sim 20 \mu \mathrm{M}\right)$. However, Phe binding to the R171AMtuDAH7PS mutant was severely compromised $\left(K_{\mathrm{d}} \gg 1000 \mu \mathrm{M}\right)$, demonstrating that site 2, where $\mathrm{R} 171$ is located, is primarily responsible for binding Phe. On the other hand, Tyr was found to bind to this enzyme variant with slightly higher affinity, consistent with the presence of an alternative site that shows a preference for the binding of Tyr relative to Phe. Despite the relatively tight binding of Tyr to the R171AMtuDAH7PS mutant, the Trp+Tyr combination did not elicit the same level of kinetic inhibition of this enzyme variant as was observed for the wild-type MtuDAH7PS, indicating that a competent site 2 is required for maximal inhibitory response to Trp + Tyr (Fig. 2).

\section{Mutation of Arg256 (at site 3) impairs Tyr binding}

Arg256 is responsible for binding the carboxylate functionality of Tyr or Phe in site 3. Site 3 was found to be occupied by Phe when high concentrations of this ligand were employed in crystal soaking experiments, and by Tyr at low concentrations. Arg256 was substituted by Ala in order to investigate the relevance of Tyr and Phe binding to site 3 . The R256AMtuDAH7PS enzyme retains sensitivity to the combination of Trp+Phe to a level comparable to that observed for the wild-type MtuDAH7PS, confirming that site 3 plays a minor role in the inhibition that arises from the binary combination of Trp and Phe (Fig. 2c). In contrast, the inhibitory response of 
Table 1. Dissociation constants measured from ITC inhibitor binding experiments

\begin{tabular}{lcccr}
\hline & & & $K_{\mathrm{d}}(\mu \mathrm{M})$ & \\
\cline { 3 - 5 } $\begin{array}{l}\text { Titrated } \\
\text { ligand }\end{array}$ & $\begin{array}{c}\text { Background } \\
\text { ligand }\end{array}$ & Wild-type MtuDAH7PS & R171AMtuDAH7PS & R256AMtuDAH7PS \\
\hline Phe & - & $21 \pm 1^{\text {a }}$ & $\gg 1000$ & $17 \pm 1$ \\
Tyr & - & $39 \pm 1^{\text {a }}$ & $19 \pm 2$ & $54 \pm 4$ \\
Tyr & Phe & $12 \pm 1$ & $40 \pm 3$ & $120 \pm 20$ \\
\hline
\end{tabular}

Background Phe concentration is $50 \mu \mathrm{M}$.

ITC data are shown in the Supplementary Material (Tables S4 and Table S5; Figs. S6-S8).

As reported by Jiao et al. ${ }^{21}$

R256AMtuDAH7PS to the combination of Trp and Tyr was essentially abolished. These observations indicate that site 3 plays a key functional role in the inhibition of MtuDAH7PS by the Trp+Tyr binary combination. Notably, the ability of Tyr to augment the inhibition of the Trp + Phe combination is lost for the R256AMtuDAH7PS variant, indicating that a competent site 3 is required for the response to all three aromatic amino acids.

ITC experiments were conducted to further probe ligand binding of the R256AMtuDAH7PS mutant. Whereas Phe binding is unaltered by this mutation at site 3 , Tyr binding is slightly impaired. These observations illustrate clearly the ability of Tyr to bind to both sites 2 and 3 of MtuDAH7PS and the preference of Phe for site 2 rather than site 3 . The observations that Tyr binds to R256AMtuDAH7PS with a reduced affinity yet more tightly to the R171AMtuDAH7PS indicate that while Tyr can bind to both sites, it has a slight preference for site 3 . Tyr binds with lower affinity to both R256AMtuDAH7PS and R171AMtuDAH7PS when the enzymes are incubated with Phe prior to titration (Table 1). This indicates that Tyr competes with Phe for binding to both sites.

In summary, the influence of mutations of sites 2 and 3 on binding constants of Phe and Tyr to the enzyme and on the inhibition by the combinations of amino acids illustrates the functional importance of these two sites. Consistent with the ligand-bound crystal structures of the enzyme using varying concentrations of ligands, site 2 is able to bind Phe or Tyr, whereas site 3 is Tyr-selective and only binds Phe at high concentrations. With respect to inhibition, maximum inhibition requires the binding of Phe in site 2 whereas binding of Tyr in this site has, remarkably, minor influence on inhibition. Site 3 is required for the Tyr-mediated augmentation of the inhibition caused by the Trp+Phe combination and the functional response of the enzyme to all three aromatic amino acids.

\section{Discussion}

Many key enzymes of biological pathways are regulated by pathway end products, such as the $\alpha$ - aminoadipate pathway that is responsible for lysine biosynthesis in fungi and certain archaebacteria, ${ }^{23}$ the biosynthetic pathway of leucine, ${ }^{24}$ and that of histidine. ${ }^{25}$ The shikimate pathway is a branched pathway with multiple pathway end products and is responsible for biosynthesis of aromatic amino acids Trp, Phe, and Tyr. While the pathway flux can be regulated in individual branches by feedback regulation of specific enzymes down the pathway, and MtuDAH7PS plays a role in this through complex formation with the $M$. tuberculosis chorismate mutase, ${ }^{22}$ there is also a need to govern the entrance of valuable starting materials into the system and limit the accumulation of reactive intermediates, by regulating the enzymes in the main trunk of the pathway. DAH7PS as the first enzyme of the shikimate pathway represents a key point for the regulation of the pathway output. However, with multiple pathway end products, the regulation of DAH7PS poses a big challenge, because the three aromatic amino acids are required in different concentrations in cellular environment. Intracellular concentrations of aromatic amino acids Phe, Trp, and Tyr in glucose-fed Escherichia coli have been reported to be $18 \mu \mathrm{M}, 12 \mu \mathrm{M}$, and $29 \mu \mathrm{M}$, respectively. ${ }^{26}$ Therefore, the regulation of DAH7PS needs to be tunable and responsive to different concentrations of the three aromatic amino acids, so that when only one type of aromatic amino acid is overproduced, the entire pathway will not be shut down and limit the supply of the other aromatic amino acids. Different organisms adopt different strategies for the regulation of this enzyme. For example, in E. coli, multiple isozymes of DAH7PS are expressed and each of the isozymes is inhibited by one kind of aromatic amino acid. However, only one DAH7PS enzyme is encoded by the $M$. tuberculosis genome, which is a relatively uncommon feature for organisms using the shikimate pathway. In order to regulate overall pathway flux, M. tuberculosis seems to have evolved a highly sophisticated regulatory mechanism that is tunable by multiple pathway end products using a single DAH7PS enzyme.

MtuDAH7PS is relatively insensitive to Trp, Phe, or Tyr alone at low concentrations; binary combinations of aromatic amino acids that include Trp (Trp+Phe 
and Trp + Tyr) can inhibit the enzyme significantly, with Trp + Phe being the most potent combination. The binary response caused by Trp + Phe is found to be augmented by the binding of Tyr and results in almost complete loss of the enzyme activity. Crystal structures of MtuDAH7PS in complex with different ligands show that there are three distinctive allosteric binding sites on the enzyme. Two of these allosteric binding sites are located at the dimer and tetramer interfaces of the enzyme, and the third site is located on the exterior surface of the enzyme near the dimer interface. Previously, it has been established that the Trp binding site is located at the tetramer interface (site 1) and that Phe can bind in both the dimer interface site (site 2) and the third site (site 3). ${ }^{20}$ Site 2 together with the Trp binding site 1 have been shown to be the sites that are responsible for the binary inhibitory response caused by the Trp +Phe combination. ${ }^{21}$ In this study, the role of site 3 and the sites responsible for the binary response of Trp + Tyr combination is clarified. The crystal structures of MtuDAH7PS in complex with Tyr and Tyr+Trp reveal that Tyr binding to site 3 may be more favorable due to additional hydrogen-bonding interactions formed between its hydroxyl moiety and the enzyme; hence, this site is identified as the selective binding site for Tyr. ITC measurements for both the wild-type and mutants of MtuDAH7PS confirm that site 3 is indeed a Tyr-selective site. Inhibition studies demonstrate that this Tyr-selective site contributes most of the inhibition response in the Tyr + Trp combination and is required for the functional response to the ternary combination of aromatic amino acids.

Allosteric regulation of protein function by multiple allosteric effectors has been reported for other protein systems. Glutamine phosphoribosylpyrophosphate amidotransferase from Bacillus subtilis catalyzes the initial reaction in de novo purine nucleotide synthesis, and it was found to be synergistically feedback-regulated by a combination of the adenine di- and guanine mononucleotide ADP and GMP. ${ }^{27}$ The crystal structure of this enzyme in complex with ADP and GMP established that ADP binds to the allosteric site whereas GMP binds to the catalytic site. ${ }^{28}$ Another example is aspartate transcarbamoylase from $E$. coli, which catalyzes the first step in the pyrimidine biosynthetic pathway and is synergistically inhibited by pyrimidine cytidine and uracil trinucleotides CTP and UTP, respectively. The binding of allosteric effectors CTP and UTP causes a change in overall conformation of the enzyme, which shifts the enzyme from the active R-state to the inactive T-state. ${ }^{29}$ Both enzymes catalyze the first reaction in a biosynthesis pathway with multiple end products and both are found to be synergistically inhibited by the pathway end products. Synergistic allostery appears to be a suitable general strategy for metabolic control of pathways with multiple end products, because the inhibitory response is enhanced only when more than one end product is being overproduced.

However, the complexity of the allosteric regulation observed for MtuDAH7PS appears to be unparalleled. There are three distinctive allosteric binding sites selective for each of the three aromatic amino acids with respect to eliciting an inhibitory response. These allosteric sites are located distant to the active site, $21 \AA$, $28 \AA$, and $24 \AA$ away from the active-site metal ion for Phe, Tyr, and Trp sites, respectively. However, despite the long distances between the ligand sites, occupancy of two or three allosteric sites operates together to communicate an inhibitory signal to the active site of the enzyme. The inhibition is fine-tuned by the number of different allosteric effectors present, providing a powerful and tunable regulatory response to cellular aromatic amino acid demands.

\section{Materials and Methods}

\section{Protein expression and purification of MtuDAH7PS and MtuDAH7PS mutants}

The allosteric binding site mutants of MtuDAH7PS were produced using the QuikChange Lightning Site-Directed Mutagenesis Kit (Stratagene) and conducted in accordance with the manufacturer's instructions. A PCR primer table is provided in the Supplementary Material, Table S6. The resulting PCR products were transformed into chemically competent One Shot TOP10 (Invitrogen) and electrocompetent BL21 (DE3) pGroESL cell lines for plasmid storage and protein expression, respectively. Protein expression and purification of both wild-type and mutant MtuDAH7PS were carried out as previously described by Webby et al. except that Thesit [polyoxyethylene(9)-lauryl-ether] was omitted from all buffers and $1 \mathrm{mM}$ tris(2-carboxyethyl)phosphine (TCEP) (Sigma) was added to all buffers. ${ }^{30}$

\section{Enzyme assays and kinetic measurements}

The kinetic parameters and measurements of the mutants were determined using standard assay conditions. ${ }^{30}$ MtuDAH7PS mutant activity was measured by monitoring the loss of PEP at $232 \mathrm{~nm} \quad(\varepsilon=$ $2.8103 \mathrm{M}^{-1} \mathrm{~cm}^{-1}$ at $303 \mathrm{~K}$ ) in the presence of E4P using a Varian Cary 1 UV-visible spectrophotometer. ${ }^{30}$ The assay solution contained PEP (fixed at $300 \mu \mathrm{M}$ or varied to determine PEP $K_{m}$ ) (Research Chemicals), E4P (fixed at $300 \mu \mathrm{M}$ or varied to determine E4P $K_{\mathrm{m}}$ ) (Sigma), and $\mathrm{MnSO}_{4}(100 \mu \mathrm{M})$ (Sigma) in assay buffer $\{50 \mathrm{mM} \mathrm{1,3-}$ bis[tris(hydroxymethyl)methylamino]propane buffer (Sigma), $\mathrm{pH} 7.5$, and $1 \mathrm{mM}$ TCEP . PEP and E4P solutions were made up in assay buffer and the $\mathrm{MnSO}_{4}$ solution was made up in ultrapure water. The reaction was initiated by the addition of purified mutant MtuDAH7PS $(\sim 2.5 \mathrm{mg} / \mathrm{mL})$. Initial reaction rates were determined by a least-squares fit of the initial-rate data. $K_{\mathrm{m}}$ and $k_{\text {cat }}$ values were determined by fitting the data to the Michaelis- 
Menten equation using the program GraFit (Erithacus software).

\section{Feedback inhibition studies}

Solutions of L-Phe (Sigma), L-Tyr (Sigma), and L-Trp (Sigma) in ultrapure water were added to standard assay reaction mixtures to give a concentration of either 100 , 200 , or $400 \mu \mathrm{M}$ in inhibitor studies. The assay solution contained PEP $(150 \mu \mathrm{M})$, E4P $(150 \mu \mathrm{M})$, and $\mathrm{MnSO}_{4}$ $(100 \mu \mathrm{M})$ in assay buffer. The reactions were initiated by adding either MtuDAH7PS or mutant MtuDAH7PS. Stead$y$-state kinetic assays were conducted in the presence of no, one, two, or all three aromatic amino acids.

\section{Isothermal titration calorimetry}

The binding of MtuDAH7PS and the allosteric binding site mutants of MtuDAH7PS to the aromatic amino acid ligands was assessed by ITC, using a VP-ITC unit operating at $298 \mathrm{~K}$ (MicroCal, GE Healthcare). Protein was purified in the binding buffer, and all solutions were degassed in a vacuum before being used. The binding buffer was $10 \mathrm{mM}$ 1,3-bis[tris(hydroxymethyl)methylamino]propane ( $\mathrm{pH} 7.5$ ), $150 \mathrm{mM} \mathrm{NaCl}, 200 \mu \mathrm{M} \mathrm{MnSO}_{4}$, $200 \mu \mathrm{M}$ TCEP, and $200 \mu \mathrm{M}$ PEP. All titrations are composed of 29 injections of ligand (one 2- $\mu \mathrm{L}$ injection followed by twenty-eight $10-\mu \mathrm{L}$ injections), except titrations of Tyr into wild-type MtuDAH7PS, which are composed of 57 injections (one 2- $\mu \mathrm{L}$ injection and fifty-six $5-\mu \mathrm{L}$ injections; Supplementary Material, Table S5).

To allow for diffusion of the ligand across the needle tip during the equilibration period, the first data point was routinely deleted. Heats of dilution experiments were measured independently and subtracted from the integrated data before curve fitting in Origin 7.0 with the standard one-site model supplied by MicroCal, except for the analysis of R171AMtuDAH7PS with Trp, which used a two-site sequential binding site model supplied by MicroCal.

\section{Structure determination}

Crystals for soaking experiments were obtained as previously described. ${ }^{30,31}$ Crystals were soaked in droplets containing appropriate concentrations of amino acids that had been pre-equilibrated against the same reservoir as the crystallization drop. Diffraction data were collected at the MX beamlines at the Australian Synchrotron. ${ }^{32}$ Structures were solved by molecular replacement using the unliganded MtuDAH7PS dimer (PDB code 3NV8) as the search model. ${ }^{33,34}$ The protein structure was developed in successive cycles of model building with Coot and refinement with REFMAC5, using intensity-based twin refinement. ${ }^{35,36}$ Structures were validated using the MolProbity server ${ }^{37}$ and TLS groups were generated by the TLSMD server. ${ }^{38} 2 F_{\mathrm{o}}-F_{\mathrm{c}}$ and $F_{\mathrm{o}}-F_{\mathrm{c}}$ omit maps of the ligand-binding sites were examined after complete refinement of the protein backbone and side chains, and ligands were modeled into appropriate density. Omit maps shown in figures were generated before addition of ligands to the model.

\section{Accession numbers}

Coordinates and structure factors have been deposited in the PDB with accession numbers 2YPO, 2YPP, and 2YPQ.

\section{Acknowledgements}

This research was undertaken on the MX beamlines at the Australian Synchrotron, Victoria, Australia. We acknowledge travel funding by the New Zealand Synchrotron Group. This research was partly funded by the Maurice Wilkins Centre for Biomolecular Discovery and the New Zealand Marsden Fund (UOC1105). N.J.B. acknowledges funding from the Maurice Wilkins Centre and University of Canterbury Doctoral Scholarships. S.R. acknowledges funding from a New Zealand International Doctoral Research Scholarship and a University of Canterbury Doctoral Scholarship.

\section{Supplementary Data}

Supplementary data to this article can be found online at http://dx.doi.org/10.1016/j.jmb.2012.12.019

Received 31 October 2012; Received in revised form 19 December 2012; Accepted 20 December 2012 Available online 28 December 2012

Keywords: shikimate pathway; 3-deoxy-D-arabino-heptulosonate 7-phosphate synthase; Mycobacterium tuberculosis; allosteric regulation; mutagenesis

Abbreviations used: DAH7PS, 3-deoxy-D-arabino-heptulosonate 7-phosphate synthase; E4P, erythrose 4-phosphate; ITC, isothermal titration calorimetry; PEP, phosphoenolpyruvate; PDB, Protein Data Bank; TCEP, tris(2-carboxyethyl)phosphine.

\section{References}

1. Boehr, D. D., Nussinov, R. \& Wright, P. E. (2009). The role of dynamic conformational ensembles in biomolecular recognition. Nat. Chem. Biol. 5, 789-796.

2. Changeux, J. P. \& Edelstein, S. J. (2005). Allosteric mechanisms of signal transduction. Science, 308, 1424-1428.

3. Goodey, N. M. \& Benkovic, S. J. (2008). Allosteric regulation and catalysis emerge via a common route. Nat. Chem. Biol. 4, 474-482.

4. Kuriyan, J. \& Eisenberg, D. (2007). The origin of protein interactions and allostery in colocalization. Nature, 450, 983-990. 
5. Smock, R. G. \& Gierasch, L. M. (2009). Sending signals dynamically. Science, 324, 198-203.

6. Monod, J., Wyman, J. \& Changeux, J. P. (1965). On the nature of allosteric transitions: a plausible model. J. Mol. Biol. 12, 88-118.

7. Koshland, D. E., Nemethy, G. \& Filmer, D. (1966). Comparison of experimental binding data and theoretical models in proteins containing subunits. Biochemistry, 5, 365-385.

8. Kern, D. \& Zuiderweg, E. R. (2003). The role of dynamics in allosteric regulation. Curr. Opin. Struct. Biol. 13, 748-757.

9. Gunasekaran, K., Ma, B. \& Nussinov, R. (2004). Is allostery an intrinsic property of all dynamic proteins? Proteins, 57, 433-443.

10. Swain, J. F. \& Gierasch, L. M. (2006). The changing landscape of protein allostery. Curr. Opin. Struct. Biol. 16, 102-108.

11. Bahar, I., Chennubhotla, C. \& Tobi, D. (2007). Intrinsic dynamics of enzymes in the unbound state and relation to allosteric regulation. Curr. Opin. Struct. Biol. 17, 633-640.

12. Tsai, C. J., del Sol, A. \& Nussinov, R. (2008). Allostery: absence of a change in shape does not imply that allostery is not at play. J. Mol. Biol. 378, $1-11$.

13. Tsai, C. J., del Sol, A. \& Nussinov, R. (2009). Protein allostery, signal transmission and dynamics: a classification scheme of allosteric mechanisms. Mol. Biosyst. 5, 207-216.

14. Ascenzi, P., Bocedi, A., Bolli, A., Fasano, M., Notari, S. \& Polticelli, F. (2005). Allosteric modulation of monomeric proteins. Biochem. Mol. Biol. Educ. 33, 169-176.

15. Ascenzi, P. \& Fasano, M. (2010). Allostery in a monomeric protein: the case of human serum albumin. Biophys. Chem. 148, 16-22.

16. Popovych, N., Sun, S., Ebright, R. H. \& Kalodimos, C. G. (2006). Dynamically driven protein allostery. Nat. Struct. Mol. Biol. 13, 831-838.

17. Bentley, R. (1990). The shikimate pathway-a metabolic tree with many branches. Crit. Rev. Biochem. Mol. Biol. 25, 307-384.

18. Roberts, F., Roberts, C. W., Johnson, J. J., Kyle, D. E., Krell, T., Coggins, J. R. et al. (1998). Evidence for the shikimate pathway in apicomplexan parasites. Nature, 393, 801-805.

19. Campbell, S. A., Richards, T. A., Mui, E. J., Samuel, B. U., Coggins, J. R., McLeod, R. \& Roberts, C. W. (2004). A complete shikimate pathway in Toxoplasma gondii: an ancient eukaryotic innovation. Int. J. Parasitol. 34, 5-13.

20. Webby, C. J., Jiao, W., Hutton, R. D., Blackmore, N. J., Baker, H. M., Baker, E. N. et al. (2010). Synergistic allostery, a sophisticated regulatory network for the control of aromatic amino acid biosynthesis in Mycobacterium tuberculosis. J. Biol. Chem. 285, 30567-30576.

21. Jiao, W., Hutton, R. D., Cross, P. J., Jameson, G. B. \& Parker, E. J. (2012). Dynamic cross-talk among remote binding sites: the molecular basis for unusual synergistic allostery. J. Mol. Biol. 415, 716-726.

22. Sasso, S., Okvist, M., Roderer, K., Gamper, M., Codoni, G., Krengel, U. \& Kast, P. (2009). Structure and function of a complex between chorismate mutase and DAHP synthase: efficiency boost for the junior partner. EMBO J. 28, 2128-2142.

23. Bulfer, S. L., Scott, E. M., Pillus, L. \& Trievel, R. C. (2010). Structural basis for L-lysine feedback inhibition of homocitrate synthase. J. Biol. Chem. 285, 10446-10453.

24. de Carvalho, L. P., Argyrou, A. \& Blanchard, J. S. (2005). Slow-onset feedback inhibition: inhibition of Mycobacterium tuberculosis alpha-isopropylmalate synthase by L-leucine. J. Am. Chem. Soc. 127, 10004-10005.

25. Alifano, P., Fani, R., Lio, P., Lazcano, A., Bazzicalupo, M., Carlomagno, M. S. \& Bruni, C. B. (1996). Histidine biosynthetic pathway and genes: structure, regulation, and evolution. Microbiol. Rev. 60, 44-69.

26. Bennett, B. D., Kimball, E. H., Gao, M., Osterhout, R., Van Dien, S. J. \& Rabinowitz, J. D. (2009). Absolute metabolite concentrations and implied enzyme active site occupancy in Escherichia coli. Nat. Chem. Biol. 5, 593-599.

27. Meyer, E. \& Switzer, R. L. (1979). Regulation of Bacillus subtilis glutamine phosphoribosylpyrophosphate amidotransferase activity by end products. J. Biol. Chem. 254, 5397-5402.

28. Chen, S., Tomchick, D. R., Wolle, D., Hu, P., Smith, J. L., Switzer, R. L. \& Zalkin, H. (1997). Mechanism of the synergistic end-product regulation of Bacillus subtilis glutamine phosphoribosylpyrophosphate amidotransferase by nucleotides. Biochemistry, 36, 10718-10726.

29. Kantrowitz, E. R. (2012). Allostery and cooperativity in Escherichia coli aspartate transcarbamoylase. Arch. Biochem. Biophys. 519, 81-90.

30. Webby, C. J., Baker, H. M., Lott, J. S., Baker, E. N. \& Parker, E. J. (2005). The structure of 3-deoxy-Darabino-heptulosonate 7-phosphate synthase from Mycobacterium tuberculosis reveals a common catalytic scaffold and ancestry for type I and type II enzymes. J. Mol. Biol. 354, 927-939.

31. Webby, C. J., Lott, J. S., Baker, H. M., Baker, E. N. \& Parker, E. J. (2005). Crystallization and preliminary Xray crystallographic analysis of 3-deoxy-D-arabinoheptulosonate-7-phosphate synthase from Mycobacterium tuberculosis. Acta Crystallogr., Sect. F: Struct. Biol. Cryst. Commun. 61, 403-406.

32. McPhillips, T. M., McPhillips, S. E., Chiu, H. J., Cohen, A. E., Deacon, A. M., Ellis, P. J. et al. (2002). Blu-Ice and the Distributed Control System: software for data acquisition and instrument control at macromolecular crystallography beamlines. J. Synchrotron Radiat. 9, 401-406.

33. Winn, M. D., Ballard, C. C., Cowtan, K. D., Dodson, E. J., Emsley, P., Evans, P. R. et al. (2011). Overview of the CCP4 suite and current developments. Acta Crystallogr., Sect. D: Biol. Crystallogr. 67, 235-242.

34. Vagin, A. \& Teplyakov, A. (1997). MOLREP: an Automated Program for Molecular Replacement. J. Appl. Crystallogr. 30, 1022-1025.

35. Emsley, P. \& Cowtan, K. (2004). Coot: model-building tools for molecular graphics. Acta Crystallogr., Sect. D: Biol. Crystallogr. 60, 2126-2132.

36. Murshudov, G. N., Skubak, P., Lebedev, A. A., Pannu, N. S., Steiner, R. A., Nicholls, R. A. et al. (2011). 
REFMAC5 for the refinement of macromolecular crystal structures. Acta Crystallogr., Sect. D: Biol. Crystallogr. 67, 355-367.

37. Chen, V. B., Arendall, W. B., III, Headd, J. J., Keedy, D. A., Immormino, R. M., Kapral, G. J. et al. (2010). MolProbity: all-atom structure validation for macromolecular crystallography. Acta Crystallogr., Sect. D: Biol. Crystallogr. 66, 12-21.

38. Painter, J. \& Merritt, E. A. (2006). TLSMD web server for the generation of multi-group TLS models. J. Appl. Crystallogr. 39, 109-111. 